\title{
Strain rate and thermal softening effects in shear testing of AA7075-T6 sheet
}

\author{
Taamjeed Rahmaan ${ }^{1}$, Ping Zhou ${ }^{1, \mathrm{a}}$, Cliff Butcher $^{1}$, and Michael J. Worswick ${ }^{1}$ \\ ${ }^{1}$ University of Waterloo, Mechanical and Mechatronics Engineering Department, Waterloo, Ontario, Canada
}

\begin{abstract}
Shear tests were performed at strain rates ranging from quasi-static $\left(0.01 \mathrm{~s}^{-1}\right)$ to $500 \mathrm{~s}^{-1}$ for AA7075T6 sheet metal alloy at room temperature. A miniature sized shear specimen was used in this work to perform high strain rate shear testing. Digital image correlation (DIC) techniques were employed to measure the strains in the experiments. At maximum in-plane shear strains greater than 20\%, the AA7075-T6 alloy demonstrated a reduced work hardening rate at elevated strain rates. At lower strains, the AA7075-T6 alloy showed mild positive rate sensitivity. The strain to localization (using the Zener-Holloman criterion), measured using the DIC technique, decreased with strain rate in shear loading. The strain at complete failure, however, exhibited an increase at the highest strain rate $\left(500 \mathrm{~s}^{-1}\right)$. The current work also focused on characterization of the thermal conditions occurring during high rate loading in shear with in situ high speed thermal imaging. Experimental results from the highest strain rate $\left(500 \mathrm{~s}^{-1}\right)$ tests showed a notable increase in temperature within the specimen gauge region as a result of the conversion of plastic deformation energy into heat.
\end{abstract}

\section{Introduction}

Vehicle weight reduction has been traditionally achieved by using advanced lightweight high strength metal alloys within automotive structures and body panels, while maintaining strength and crash performance. High strength aluminum alloys (such as 7000 -series aluminum) can provide the same level of strength as conventional steel components but with higher weight reduction capability due to the lower density of aluminium [1]. In automobile crash events or high speed metal forming, the local strain rates can vary from quasi-static conditions up to $10^{3} \mathrm{~s}^{-1}$ strain rate. Understanding the constitutive response and fracture mechanisms associated with dynamic loading of these materials under different loading and strain rate conditions is required to further increase their application within the automotive industry.

Large plastic strains are often involved in the sheet metal forming processes. An optimized forming technique can be achieved with the accurate knowledge of the elastoplastic behaviour of the metals until failure. Typically, uniaxial tensile tests are used for high strain rate characterization of sheet metals until the onset of necking. Such testing method limits the accurate measurements of the material behaviour to relatively small strains due to onset of diffuse or localized necking. The material response can be investigated for much larger plastic strains through shear tests since localized necking does not occur and plane stress conditions are satisfied until fracture. A conversion of shear data to tensile data can be useful to obtain flow stress curves for larger strains [2]. In addition, the fracture characteristics of a material under shear stress states has significant importance in developing stress state-dependent fracture surfaces and damage models to predict failure in automotive crash simulations [3-4].

At larger strain rates $\left(>1 \mathrm{~s}^{-1}\right)$, deformation heating becomes prominent at larger strain levels, and consequently has the potential to result in thermal softening [5-6]. An isothermal assumption during plastic deformation is valid in the case of low strain rate experiments since the heat generated within the specimen is dissipated either through conduction to the grip regions or through convection to air [7]. Conditions become more adiabatic at high strain rates since the flow stress is higher and the heat generated cannot be dissipated immediately [8-9]. Rahmaan et al. [2] conducted shear tests on AA5182-O sheet metal alloys, and demonstrated a decrease in strain to localization with strain rate due to the effect of temperature rise. A similar response was observed by Pérez-Castellanose et al. [10] for an AA6082 alloy where the specimen temperature increased with an increase in strain rate under dynamic compression loading. El-Magd and Abouridouane [11] also demonstrated that the flow curves at quasi-static condition increased continuously with increasing strain due to strain hardening, while at higher strain rates $(\geq$ $1000 \mathrm{~s}^{-1}$ ) the flow curves increased only at lower strains and decreased beyond a stress maximum due to thermal softening caused by the adiabatic temperature rise in the specimen. Currently, only a limited number of studies are available on the effect of strain rate on the constitutive and fracture response of 7000-series aluminum sheet alloys under shear loading.

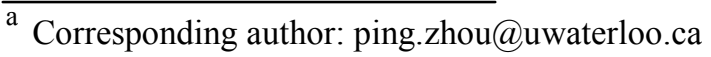


In the present work, the shear deformation behaviour and fracture characteristics of AA7075-T6 sheet metal alloy have been investigated at strain rates of $0.01,10$, and $500 \mathrm{~s}^{-1}$. The focus herein is on the temperature rise due to plastic work with increase in strain rate and its effect on flow stress and strain to failure in shear.

\section{Experiments}

\subsection{Materials}

The material studied was AA7075-T6 in nominal 2 mm thick sheets. This material was selected due to its importance in lightweight automotive applications. Specimens were machined along the sheet diagonal $\left(45^{\circ}\right.$ to rolling) direction.

\subsection{Specimen Geometry}

The micro-shear specimen geometry [2], developed based on the mini-shear specimen geometry of Peirs et al. [12], was used in this work. Abedini et al. [13-14] have shown that the mini-shear geometry can be used for both constitutive and fracture characterization of sheet metal alloys due to its relatively homogeneous shear zone and avoidance of through-thickness machining. The microshear specimen has been validated by Rahmaan et al. [2] for use in high strain rate shear testing. The geometry of the micro-shear specimens is shown in Figure 1.

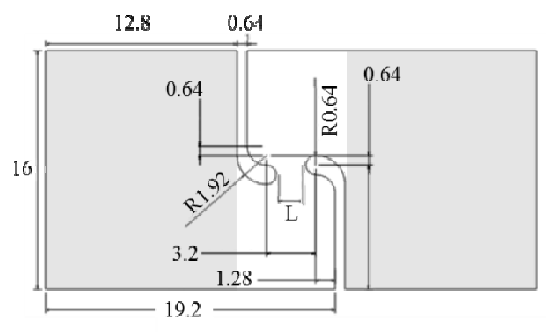

Fig. 1. Shear specimen geometry. Dimensions are in $\mathrm{mm}$. Shaded regions are the grip area.

\subsection{Apparatus and Procedure}

A servo-mechanical MTS Criterion Model 45 testing frame was used to conduct shear testing at nominal von Mises equivalent strain rates of $0.01 \mathrm{~s}^{-1}$ (or quasi-static). Cross-head velocities for the experiments were 0.019 $\mathrm{mm} / \mathrm{s}$ for the micro shear specimen testing.

A Hydraulic Intermediate Strain Rate (HISR) apparatus, developed at the University of Waterloo, was used to perform shear tests at strain rates of 10 and $500 / \mathrm{s}$. The reader is referred to [15] for a detailed description of the HISR apparatus. Cross-head velocities of approximately $15 \mathrm{~mm} / \mathrm{s}$ and $1100 \mathrm{~mm} / \mathrm{s}$ were used to achieve the nominal strain rates of 10 and $500 \mathrm{~s}^{-1}$, respectively.

The strains were measured using digital image correlation (DIC) techniques. Point Grey Research GRAS-50S5M-C cameras were employed for the quasistatic condition and Photron SA5 high speed cameras for the higher strain rates. In the present work, the frame rates (frames per second, fps) and corresponding image sizes were: $10 \mathrm{fps}(2048 \times 2048$ pixels $), 5,000 \mathrm{fps}(576 \mathrm{x}$ 304 pixels), and 262,500 fps ( $128 \times 128$ pixels) for strain rates of $0.01,10$, and $500 \mathrm{~s}^{-1}$, respectively. Full-field logarithmic (true) strain measurements were obtained using Vic-3D software from Correlated Solutions Inc. In the current work, the maximum in-plane shear strain $\left(\varepsilon_{12}^{\max }\right)$ was adopted as the measure of deformation since it uniquely defines the state of strain from small to large strain levels whereas the applied shear strain $\left(\varepsilon_{12}\right)$ cannot [2]. The maximum in-plane shear strain for simple shear deformation is calculated using Eq. (1), where the major principal strain, $\varepsilon_{11}$, and minor principal strain, $\varepsilon_{12}$, can be obtained directly through the DIC measurements.

$$
\varepsilon_{12}^{\max }=\sqrt{\varepsilon_{11}^{2}+\varepsilon_{12}^{2}}
$$

Rahmaan et al. [2] showed that the selection of step and filter size used in the DIC analysis has notable effect on the reported values of strain for particular imaging conditions. Such an effect is described by the term, Virtual Strain Gauge Length (VSGL), also defined in Eq. (2). The VSGL of 0.3 was selected for the current work as it was found to provide the most accurate results for the micro-shear specimens [2].

$$
V S G L=R O I \times S S \times F S
$$

where, $R O I$ is the resolution of the area of interest (measured as $0.033 \mathrm{~mm} /$ pixel), $S S$ is the step size (taken as 1), and $F S$ is the filter size (taken as 9). As discussed in Peirs et al. [12] and Abedini et al. [13], the average shear stress, $\tau_{\text {avg }}$, can be computed as:

$$
\tau_{\text {avg }}=\frac{F}{A_{\text {shear }}}=\frac{F}{L t}
$$

where $F$ is the force, $t$ is the thickness, and $L$ is the length of the shear region with nominal values of $1.9 \mathrm{~mm}$ for the micro-shear sample. The as-fabricated gauge length of each sample was measured using an optical microscope, while the thickness was measured using a precision micrometer. Three to five repeat tests were performed for each test condition and an average stress-strain curve was generated by interpolating the test data using maximum in-plane shear strain increments of 0.0005 .

A Telops IRC-TS series high speed infrared camera was utilized for measuring the temperature changes on the specimen generated due to plastic deformation in the experiments at strain rates of 10 nad $500 \mathrm{~s}^{-1}$. The frame rates and corresponding size of the images were: $5000 \mathrm{fps}$ (128 $\times 128$ pixels) and 40,000 fps (64 × 24 pixels) for strain rates of 10 and $500 \mathrm{~s}^{-1}$, respectively.

\section{Results and Discussion}

\subsection{Shear stress-strain behaviour}


Under simple shear loading, the ratio of minor and major strain $\left(\varepsilon_{2} / \varepsilon_{1}\right)$, or in other words the principal strain ratio, is -1 . In order to validate whether a simple shear state was maintained throughout the deformation of the micro-shear samples at all strain rates considered, strain paths were obtained at the centre of the shear band. The measured strain paths are plotted in Figure 2 indicating that a constant simple shear strain state was maintained until failure and demonstrates a good agreement with the theoretical strain path.

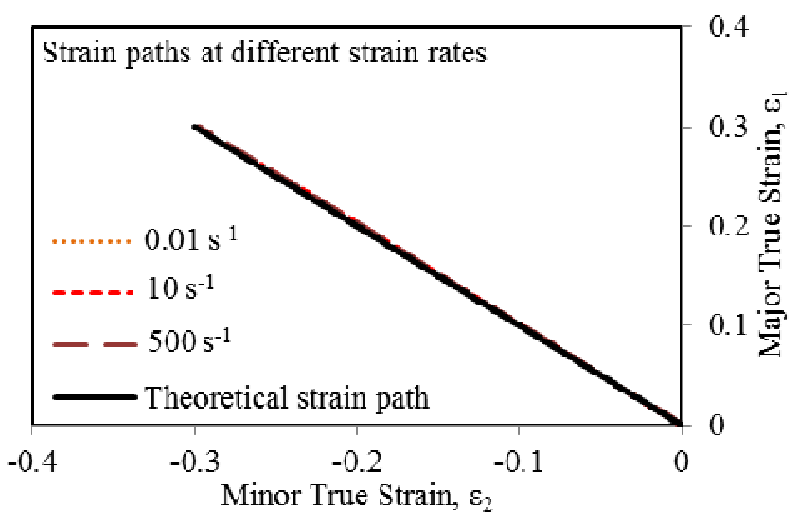

Fig. 2. Strain path obtained at the centre of the shear zone in the micro-shear specimens for AA7075-T6 at strain rates ranging from 0.01 to $500 \mathrm{~s}^{-1}$.

The repeatability of the higher rate experiments was also examined, and Figure 3 presents the shear stressstrain response for the specimens tested at $500 \mathrm{~s}^{-1}$. The standard deviation in plastic shear stress region was approximately $6 \mathrm{MPa}$. The variability was considered rather low and was typical of the results obtained for the other strain rates considered. The experimental results in this work are therefore presented in terms of the median stress-strain curve for each rate for simplicity.

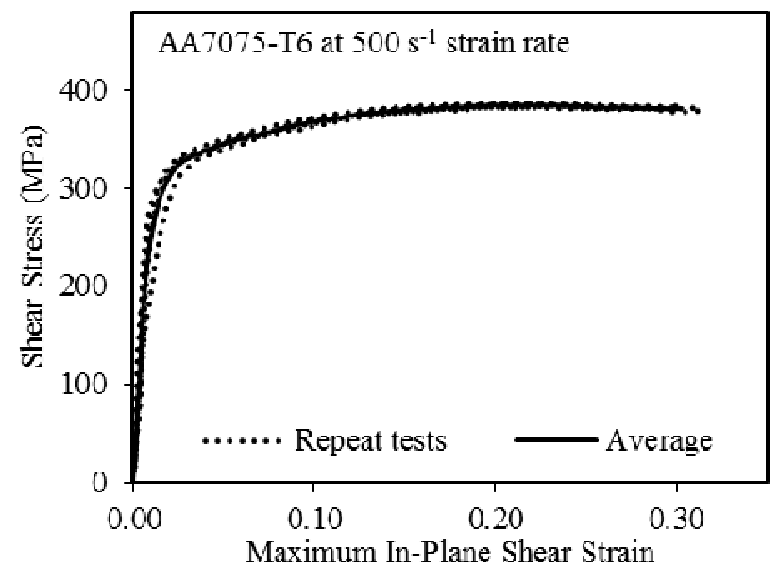

Fig. 3. Repeatability of micro-shear specimen geometry for AA7075-T6 sheet material at a strain rate of $500 \mathrm{~s}^{-1}$.

The effect of strain rate on the shear stress-strain response for the AA7075-T6 sheet materials is shown in Figure 4. The shear stress at yield and subsequent hardening response for maximum in-plane shear strains up to approximately $20 \%$ are seen to mildly increase with increasing strain rate (mild positive rate sensitivity).
Beyond this strain level, the slope of the shear stressstrain curve decreases with increased strain rate. For shear strains above $22 \%$, the shear specimens display mild negative rate sensitivity with lower stresses. In order to further demonstrate the strain rate sensitivity of AA7075-T6, the shear stress-strain was converted to equivalent stress and equivalent plastic strain (as shown in Figure 5). An equivalent plastic work based conversion methodology described by Rahman et al. [2] was followed in this work to obtain equivalent stress-strain data from measured shear data. The average shear stress ratio (the ratio of flow stress in shear to flow stress in uniaxial tension at the same level of plastic work) were found to be approximately 0.58 for AA7075-T6 and used for converting the entire shear stress-strain curves.

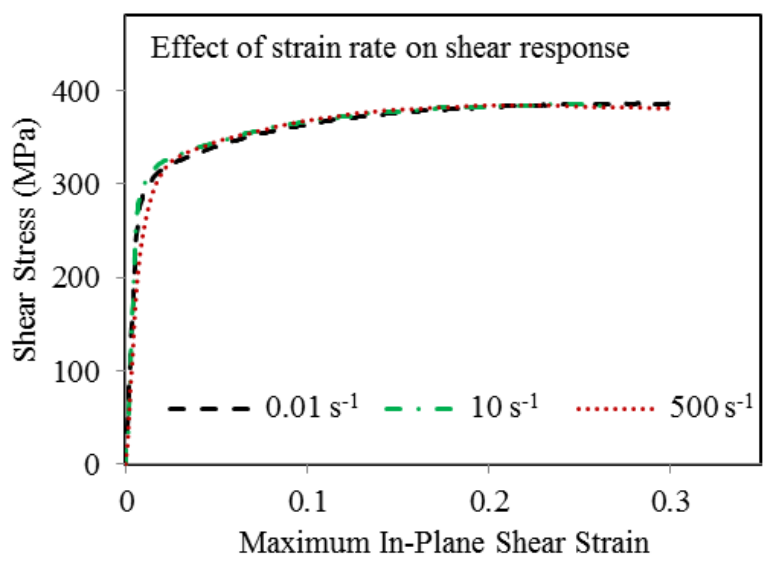

Fig. 4. Averaged shear stress response for AA7075-T6 sheet specimens at room temperature and strain rates of $0.01,10$, and $500 \mathrm{~s}^{-1}$

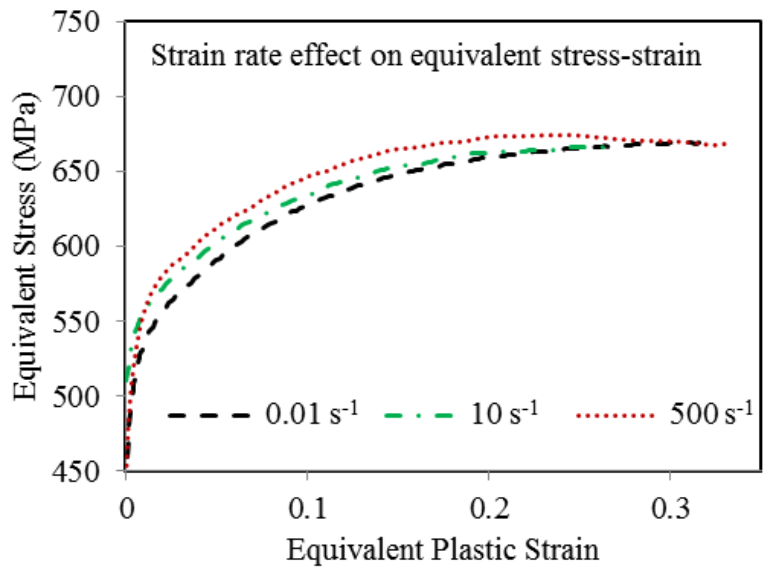

Fig. 5. Averaged equivalent stress-strain response for AA7075T6 sheet specimens at room temperature and strain rates of $0.01,10$, and $500 \mathrm{~s}^{-1}$.

The rate sensitivity of the alloy is further explored in Figure 6 which plots shear stress at constant shear strain as a function of strain rate (von Mises). Logarithmic trend lines were fit to the data points, suggesting mild positive rate sensitivity at an in-plane maximum shear strain level of $10 \%$. As the strain levels increase, the rate sensitivity drops at higher $\left(500 \mathrm{~s}^{-1}\right)$ strain rates, as reflected by the reduced slope for maximum in-plane shear strains. The 
negative strain rate sensitivity in shear stress can be explained by the temperature induced softening due to the conversion of plastic deformation energy into heat.

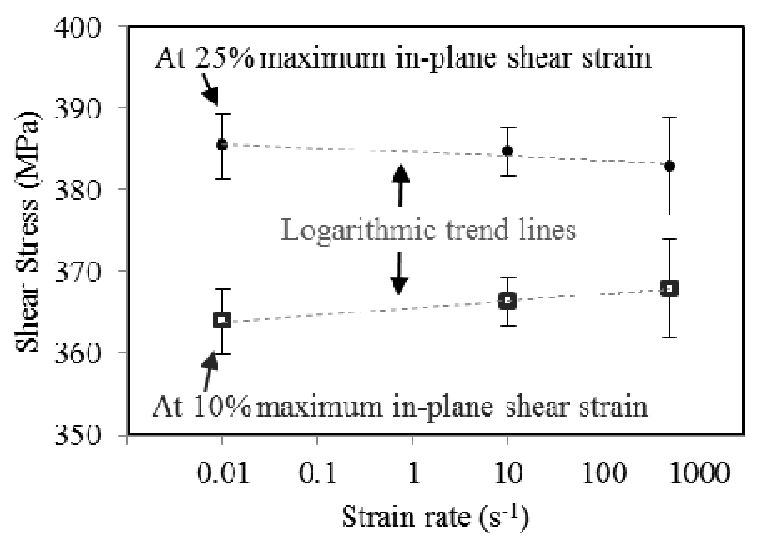

Fig. 6. Effect of von Mises equivalent strain rate on the shear strength at a maximum in-plane strains of 5\% and $25 \%$ for AA7075-T6 sheet.

\subsection{Heat generation due to plastic deformation}

A simultaneously occuring process of strain hardening and thermal softening is responsible for governing the deformation of materials due to conversion of a large fraction of deformation energy into heat. The deformation process is dominated by strain hardening at the onset of plastic deformation. However, strain hardening is reduced as deformation proceeds since thermal softening begins to dominate the deformation process. As a result, the flow stress is decreased with increasing strain at higher strain rate [16].

In the present work, the heat generation on the specimen surface during plastic deformation was analyzed with infrared technique for the strain rates of 10 and $500 \mathrm{~s}^{-1}$. One surface of the specimen was painted black to create a surface closer to a blackbody. Infrared cameras are typically calibrated in radiometric temperature rather than the true temperature of the objects in the scene. To retrieve the thermodynamic temperature, the emissivity of AA7075-T6 specimen with black paint was measured using a Gier-Dunkle DB-100 reflectometer and found to be 0.73 . The net rate of radiation heat transfer from the surface, expressed per unit area of the surface, is:

$$
q_{\text {rad }}=\in \sigma_{S B}\left(T_{s}^{4}-T_{\text {sur }}^{4}\right)
$$

where, $\epsilon$ is the emissivity, $\sigma_{S B}$ is the Stefan-Boltzmann constant, $T_{S}$ is the surface temperature of the specimen, and $T_{\text {sur }}$ (taken as $23^{\circ} \mathrm{C}$ ) is the temperature of surroundings. Assuming $q_{\text {rad }}$ is constant for the measured points in a given pixel and setting up the original emissivity to 1 (for blackbody), the measured temperatures can be adjusted by maintaining the same flux using:

$$
T_{s, a d j}=\sqrt[4]{T_{s u r}^{4}+\frac{1}{\epsilon}\left(T_{s, b}^{4}-T_{s u r}^{4}\right)}
$$

where, $T_{s, a d j}$ is the adjusted (thermodynamic) surface temperature of the specimen, $T_{s, b}$ is the radiometric surface temperature of the specimen measured using high speed infrared camera.

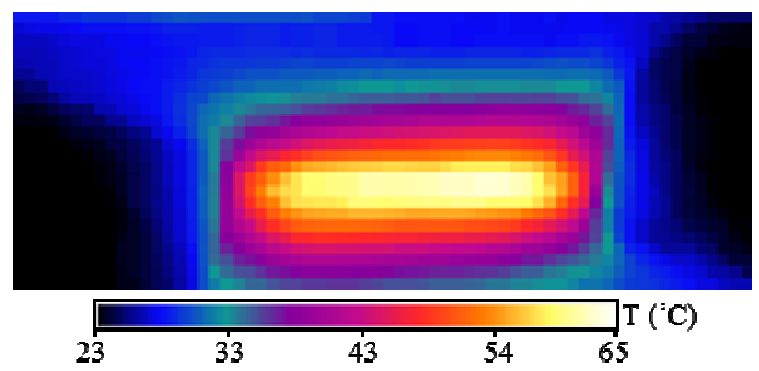

Fig. 7. Contour plot of the radiometric temperature showing temperature rise on a deformed AA7075-T6 shear specimen for a strain rate of $500 \mathrm{~s}^{-1}$ test condition.

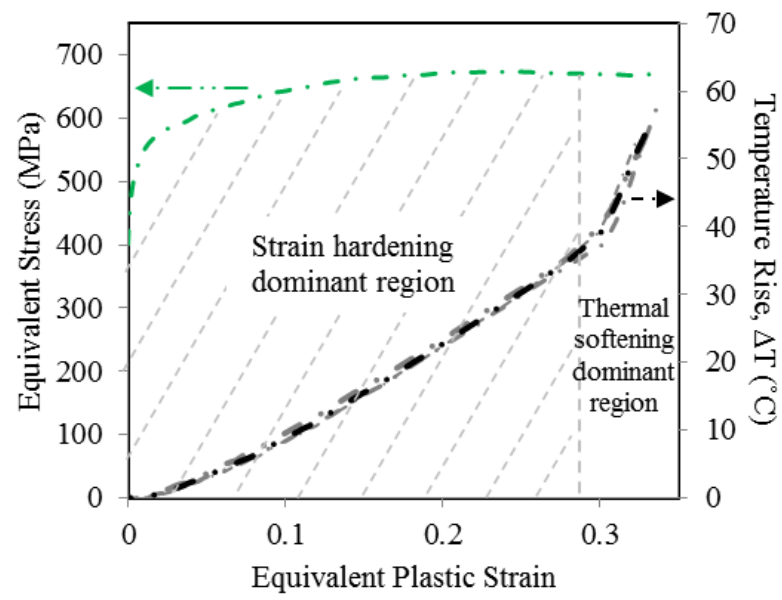

Fig. 8. Temperature increase and equivalent stress evolution for AA7075-T6 sheet material during plastic deformation for a strain rate of $500 \mathrm{~s}^{-1}$.

Figure 7 shows the observable temperature changes acquired from infrared images of AA7075-T6 sheet alloy deformed at a strain rate of $500 \mathrm{~s}^{-1}$. The temperature gradient is symmetrically distributed in the sample gauge region where the highest temperature is localized near the center of the sample gauge. The equivalent stress and temperature rise are plotted as a function of equivalent plastic strain Figure 8 for a strain rate of $500 \mathrm{~s}^{-1}$; the data is plotted up to the onset of failure. The figure shows that the increase of temperature is associated with the stress level during deformation. Up to approximately $22 \%$ equivalent plastic strain, the recorded temperature increase is approximately $23^{\circ} \mathrm{C}$, beyond which the stress saturation occurs. The increase of temperature is more rapid close to the onset of failure. As a result, the plastic deformation process was dominated by thermal softening rather than strain hardening. The final temperature increase at sample fracture was found to be approximately $57{ }^{\circ} \mathrm{C}$. Such a rapid temperature rise can account for the observed decrease in shear stress at $500 \mathrm{~s}$ ${ }^{1}$ (corresponds to Figure 4 and 5). In comparison with the behaviour at $500 \mathrm{~s}^{-1}$, the rate of temperature rise was found to be much lower at $10 \mathrm{~s}^{-1}$ (as shown in Figure 9). 
The maximum temperature rise at $10 \mathrm{~s}^{-1}$ was measured to be approximately $12^{\circ} \mathrm{C}$.

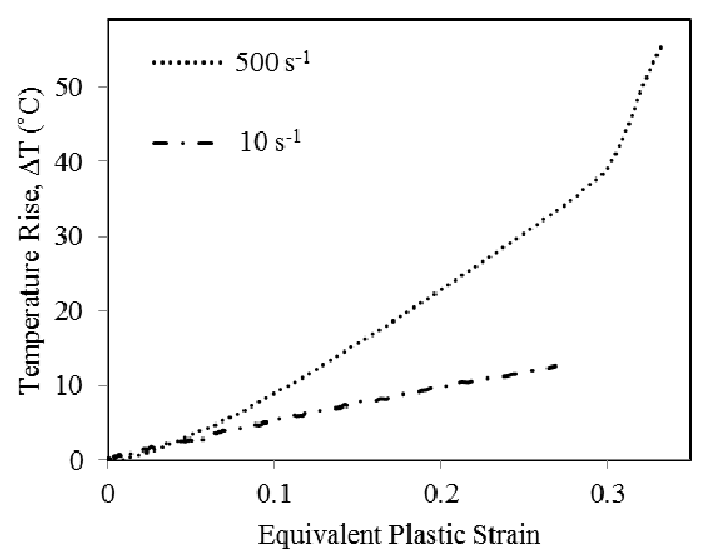

Fig. 9. Comparison of temperature rise for AA7075-T6 at strain rates of 10 and $500 \mathrm{~s}^{-1}$.

\subsection{Fracture response}

The fracture strains of AA7075-T6 alloy sheet were obtained for the range of strain rates from the measured experimental data. The onset of shear localization can be detected when the hardening capacity is exhausted according to the Zener and Hollomon [17] condition:

$$
\frac{d \sigma_{12}}{d \varepsilon_{12}^{\max }}=0
$$

The effect of strain rate on the localization strain for AA7075-T6 sheet is shown in Figure 10. For an increase in strain rate from 0.01 to $500 \mathrm{~s}^{-1}$, the von Mises equivalent strain at localization decreased from $35 \%$ to $26 \%$. A similar response was also reported by Rahmaan et al. [2] for AA5182-O and DP600, as well as by Roth and Mohr [18] for 22MnB5 steel sheet. The decrease in localization strain with increasing strain rate is attributed to the heat generated by plastic deformation, since the temperature rise can reduce the net hardening rate.

The strain to complete failure (or visible fracture) is also shown in Figure 10. The effect of strain rate on the failure strain is found to be rather interesting. For an increase in strain rate from 0.01 to $10 \mathrm{~s}^{-1}$, the von Mises equivalent strain at failure decreased from approximately 0.34 to 0.31 ( $3 \%$ decrease), which also coincides with the decrease in strain at onset of localization. However, the von Mises equivalent failure strain increased between strain rates of 10 and $500 \mathrm{~s}^{-1}$. Rahmaan et al. [2] showed that the local strain within the gauge region of the specimen increases with increasing strain rate and ultimately collapses into smaller shear bands. Such phenomena cannot be captured using the DIC method due to the limited inherent resolution. A similar spatial limitation also exists in the high speed thermal imaging method. Therefore, it is possible that at the strain rate of $500 \mathrm{~s}^{-1}$, the large strains associated with the shear band collapse would result in a temperature high enough to increase the ductility and eventually increase the failure strain. The observed change in failure strain of the materials is therefore thought to be due to either local temperature rise or strain localization within the microstructure leading to damage and failure. Understanding of such behavior requires further experimental analysis and therefore was left for future research.

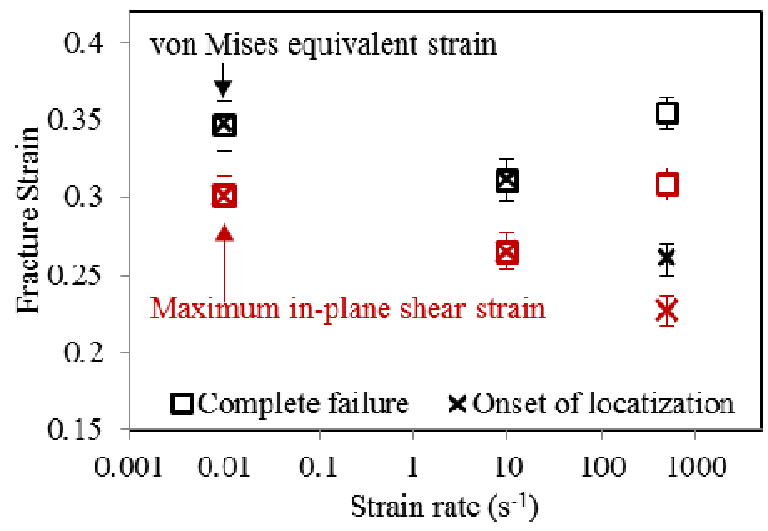

Fig. 10. Strain rate dependence of the maximum in-plane shear strain and von Mises equivalent strain at onset of localization and failure for AA7075-T6 sheet.

\section{Conclusions}

I. At maximum in-plane shear strains strains lower than approximately 0.22 , AA7075-T6 exhibited positive rate sensitivity. However, the hardening rate decreased at larger strains as the strain rate increased. The drop in rate sensitivity with strain as strain rate is increased is attributed to the increased temperature rise with strain rate as the deformation becomes more adiabatic.

II. The temperature rise associated with plastic deformation under shear loading is measured to be $57{ }^{\circ} \mathrm{C}$ for the highest strain rate $\left(500 \mathrm{~s}^{-1}\right)$ experiments, while the measured termperature rise is found to be lower $\left(12{ }^{\circ} \mathrm{C}\right)$ for tests performed at a strain rate of $10 \mathrm{~s}^{-1}$.

III. For an increase in strain rate from 0.01 to $500 \mathrm{~s}^{-1}$, the maximum in-plane shear strain to localization decreased from approximately 0.30 to 0.23 , while the failure strains of AA7075-T6 at 0.01 and $500 \mathrm{~s}^{-1}$ was found to be comparable.

The authors greatly appreciate financial support from Honda R\&D Americas, Promatek Research Centre (Cosma International), Arconic Ground Transportation Group, the Natural Sciences and Engineering Research Council of Canada, the Canada Research Chairs Secretariat, and the Ontario Research Fund.

\section{References}

1. Quadrennial technology review 2015, Chapter 8, U S Department of Energy (2015)

2. T. Rahmaan, A. Abedini, C. Butcher, N. Pathak, M. Worswick, Int. J. Impact Eng, 108, 303-321 (2017) 
3. Y. Bai, T. Wierzbicki, Int. J. Impact Eng, 24, 10711096 (2008)

4. Y. Bai, T. Wierzbicki, Int. J. Fract, 161, 1-20 (2010)

5. J. Zhang, H. Di, X. Wang, Y. Cao, J. Zhang, T. Ma, Mat. \& Design, 44, 354-364 (2013)

6. L. Cheng, X. Xue, B. Tang, D. Liu, J. Li, H. Kou, J. Li, Mat. Sc. and Eng: A, 606, 24-30 (2014)

7. B.H. Lee, N.S. Reddy, J.T. Yeom, C.S. Lee, J. Mater. Process. Tech, 187-188, 766-769 (2007)

8. A.C. Thompson, Master's thesis, University of Waterloo, Waterloo, Canada (2006)

9. S.B. Pinlung, Master's thesis, University of Windsor, Windsor, Canada (2015)

10. J.-L. P'erez-Castellanos, A. Rusinek, J. Theor. Appl. Mech, 50, 377-398 (2012)

11. E. El-Magd, M. Abouridouane, Int. J. Impact Eng, 32, 741-758 (2006)
12. J. Peirs, P. Verleysen, W. Van Paepegem, J. Degrieck, Int. J. Impact Eng, 38, 406-415 (2011)

13. A. Abedini, C. Butcher, D. Anderson, M.J. Worswick, T. Skszek, SAE World Congress, Paper \# 2015-15H-0274, (2015)

14. A. Abedini, C. Butcher, M.J. Nemcko, M.J. Worswick, Int. J. Mech. Sci, 128-129, 54-69 (2016)

15. A. Bardelcik, PhD Thesis, University of Waterloo, Waterloo, Canada (2012)

16. A.G. Odeshi, S. Al-ameeri, M.N. Bassim, J. Mater. Process. Tech, 162-163, 385-391 (2005)

17. C. Zener, J. H. Hollomon, J. App. Phy, 15, 22-32 (1944)

18. C.C. Roth, D. Mohr, EPJ Web of Conferences, 94, 01078 (2015) 